\title{
Zur Familie des Erzbischofs Heribert von Köln
}

\author{
Von \\ HERIBERT MULLER
}

\begin{abstract}
Werner Bornheim gen. Schilling, der sich als Landeskonservator von Rheinland-Pfalz vor allem mit Forschungen zu den rheinischen Höhenburgen einen Namen gemacht hat, veröffentlichte in den letzten Jahren mehrere genealogische Beiträge zur Familie der "Heribertiner" ${ }^{\prime}$ ). Die Anfänge dieses Geschlechts sieht er bei Charibert, jenem um 695 verstorbenen Grafen im Gau von Laon, dessen unbekannter Vater - vielleicht ein merowingischer Seitenverwandter ${ }^{2}$ ) - an der oberen Mosel und Maas beheimatet gewesen sein soll. Da der Graf keine männlichen Erben hinterließ (seine Tochter Berta, Bertrada vermählte sich mit dem arnulfingischen Hausmeier und späteren König Pippin d. Jüngeren), traten Haribert und Theuderich, die Enkel von Bernarius und Chrodelind, einer Schwester von Chariberts Mutter Berta, in seine Nachfolge ein. Bis zum 12. Jahrhundert sollte dann in dieser Sippe Charibert (Haribert, Heribert, Erembert u. ä.) als "Leitname" fortleben. Spuren der weitverzweigten Familie finden wir, so Bornheim, von Westfalen bis nach Südfrankreich; ihr Zentrum blieb jedoch das Land an Maas und oberer Mosel, der Eifel-/Ardennenraum ${ }^{3}$ ).
\end{abstract}

1) a) Charibert von Laon und die Prümer Stifterfamilie, in: Trierer Zs. 38 (1975), S. 113-134. Die Ergebnisse dieses Aufsatzes bilden Rahmen und Voraussetzung für das Verständnis der Einzelprobleme abhandelnden Arbeiten:

b) Zur Familie Erzbischof Heriberts von Köln, in: Jb. zur Geschichte von Stadt und Landkreis Kaiserslautern 12/3 ( Festschr. F. L. Wagner) (1974/5), S. 41-55;

c) Die Familienbeziehungen des heiligen Norbert. Heribertiner und Norbertiner, in: Jb. f. westdt, Landesgesch, 4 (1978), S. 37-60.

Eine kurze "Vorab-Zusammenfassung findet sich in Fédération archéologique, historique et folklorique de Belgique, $42 \mathrm{e}$ session (Congrès de Malmédy); Brüssel-Malmédy 1972, S. 56 f.

2) Vgl, hierzu aber schon Eduard Hlawitschka, Merowingerblut bei den Karolingern?, in: Adel und Kirche. Festschr: Gerd Tellenbach, Freiburg-Basel-Wien (1968), S. $69 \mathrm{ff}$.

ग) Dies nach: Charibert von Laon, S. 113-134. 
I.

Im Rahmen seiner Untersuchungen hat er sich in einer eigenen Arbeit "Zur Familie Erzbischof Heriberts von Köln" geäußert, deren Thesen Gegenstand dieser Miszelle sind ${ }^{4}$ ). Zuerst führt er einige Indizien an, die den Kölner Oberhirten als Mitglied des Konradinergeschlechtes erscheinen lassen (S. 42/3). Hierfür beruft er sich auf J. Jacobi, der im Rahmen seiner (fehlerhaften) Veröffentlichung über "kaiserlich-sächsische Reichs- und herzoglichfränkische Stammespolitik (936-1024)" allendings nur ein einziges Mal am Rande von "dem Konradiner Erzbischof Heribert von Köln ${ }^{4}$ sprach ${ }^{5}$ ). Als erster hat $\mathrm{m}$. W. jedoch schon Gottfried Wilhelm Leibniz im Torso seiner Geschichte des braunschweigischlüneburgischen Fürstenhauses ( ${ }_{n}$ Annales Imperii occidentis Brunsvicenses") diese Zusammenhänge bemerkt; in seiner Nachfolge griff dann der 1748 als Archivar und Bibliothekar nach Hannover berufene Christian Ludwig Scheid(t) die Ausführungen auf, die indes danach wieder in Vergessenheit gerieten \%). B. selbst stütat die Konradinerthese mit dem Hinweis auf ein Besitztum des Klosters Deutz in Ehrenbreitstein ( ${ }_{1}$ Herembrechstein") - im Schnittpunkt dreier konradinischer Gaue gelegen -, das dem Erzbischof von einem Erembert zugeeignet wurde \%). Sodann führt er die (schon bei Leibniz erörterte) Heirat des konradinischen Wetterau- und Rheingau- (nicht Oberrhein!) Grafen Udo I. (\$ 949) mit einer Tochter Heriberts I. von Vermandois an, dessen Familie sich direkter Karolingerdeszendenz rühmen konnte. Seit jener Vermählung findet sich der Name Heribert im gebhardinisch-wetterauischen Konradinerzweig ${ }^{8}$ ). Ohne diesen - mit meinem eigenen

\footnotetext{
1) Siehe Anm. 1 b.

5) In: Jb. f. Gesch. u, Kunst des Mittelrheins u. seiner Nachbargebiete $8 / 9(1957)$, S. 10.

9) a) Gottfried Wilhelm Leibniz, Gesammelte Werke, hg. v. G. H. Pertz, I, Geschichte 2/3, Hannover 1845/6 (ND 1966), Bd. 2, S. 573 Bd, 3, S. 615, 728, 803;

b) Origines Guelficae ... Opus ... coeptum a viris illustribus ... ad finem perduxit atque edidit Christianus Ludovicus Scheidius, t. 4 , Hannover 1753, S. $287-290$.

7) Rheinisches Urkundenbuch. Altere Urkunden bis 1100. Erste Lieferung: Aachen-Deutz, bearb. v. Erich Wisplinghoff, Bonn 1972 ( $=$ Publikationen der Gesellschaft f. Thein. Gesch.kunde, Bd. 57), n, 131 (S, 194).

"Herembrechstein" : Besitzliste im "Codex Theoderici Aeditui Tuitiensis", die der von B. fälschlich als Chronist bezeichnete Küster Dietrich kurz vor 1168 erstellte: Archiv f. die Gesch. des Niederrheins V/2 (1866),
S. 270 .

8) Hierzu Heribert Müller, Heribert, Kanzler Ottos III. und Erzbischof von Köln, Köln 1977 ( $=$ Veröffentlichungen des Köln. Geschichtsvereins,
Bd. 33), S. 55.
} 
Versuch weitgehend übereinstimmenden - Gedanken weiter nachzugehen, erklärt B. überraschend-abrupt: "Wie nun aber Erzbischof Heribert mit diesem [Udos Sohn Graf Heribert von der Wetterau] verwandt ist, das steht dahin" (S. 43). Die wichtige Frage, ob eine Zugehörigkeit des Kölners zur Familie der Konradiner nicht neue reichsgeschichtliche Zusammenhänge eröffnet, müßte indes noch genauer zu verfolgen sein, da die mehr genealogisch angelegte Arbeit ihre Ergebnisse kaum in diesen größeren Rahmen stellt. $\mathrm{Ob}$ meine Behauptung einer konradinischen $\mathrm{Ab}$ kunft des Erzbischofs tragfähig ist, wird der weitere Gang der Forschung entscheiden müssen; jedoch bietet sie gegenüber der noch darzulegenden These B.s den Vorteil, sich gut in die politische "Gesamtlandschaft" der Zeit einzufügen und ein wenig zu ihrer Klärung beizutragen.

Uberdies sind einige sachliche Unrichtigkeiten und Einzelfehler zu korrigieren, die sich teilweise aus einer Unkenntnis der Literatur, teilweise aus dem weitgehenden Verzicht auf das Studium der Quellen (er stützt sich fast ausschließlich auf Oedigers

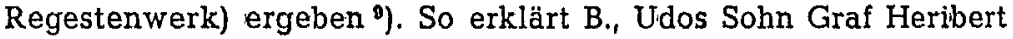
von der Wetterau ( 992) könne nicht der Erbauer der Burg Ehrenbreitstein sein - ein Blick in die von ihm an anderer Stelle zitierten einschlägigen lokalgeschichtlichen Publikationen (Michel, Jacobi, Gensicke) beweist das Gegenteil. Wenn er diesen Grafen Heribert dann richtig mit jenem "Hernbertus comes palatinus" identifiziert, der 959 als Zeuge in einer Beschreibung des Zehntsprengels der Humbach-Montabaurer Pfarrei auftritt ${ }^{10}$ ), so ist das die Erkenntnis der eben genannten Autoren, dazu vor allem I. Dietrichs, deren wichtigste Arbeit, „Das Haus der Konradiner $\left.{ }^{11}\right)$, allerdings nicht zitiert wird. B. muß sich fragen lassen, woher er um den bisher der Forschung unbekannten Namen "Gerbirch" der Mutter ides Grafen Heribert, also der Tochter des Herrn von Vermandois, weiß. Eine Verwechslung mit der gleichnamigen Gemahlin des Markgrafen Heinrich von Schweinfurt ( $\Psi$ 1017), die wahrscheinlich 'der Konradinersippe angehörte (so Schenk von Schweinsberg und Renn), scheidet wohl aus, da er auf den ostfränkischen Konradinerkreis nicht zu sprechen kommt ${ }^{12}$ ).

๑) Aus diesem Grunde werden auch in den Anmerkungen mehrfach Arbeiten zitiert, die für das Thema von Wichtigkeit sind und allgemein bekannt sein dürften, sich bei $\mathrm{B}$. jedoch nicht finden.

10) Druck: Urkundenbuch zur Geschichte der ... mittelrheinischen Territorien, Bd. 1, hg. v. Heinrich Beyer, Koblenz 1860, n. 204 - Neuere Literatur zu dieser Urkunde bei H. Müller, S. 63 A. 94.

11) Das Haus der Konradiner. Untersuchungen zur Verfassungsgeschichte der späten Karolingerzeit, Diss. (Masch.-schr.) Marburg 1952.

18) Näheres hierzu bei H. Müller, S. $66 \mathrm{f}$. 
Er erwähnt auch nicht des Erzbischofs frühere Zuordnung zu den Grafen von Rothenburg oder zum Hause Leiningen; Zuordnungen, die zum Teil über Jahrhunderte hin tradiert wurden und entsprechende Prägekraft besaßen ${ }^{13}$ ). Der einzige Hinweis, schon im Mittelalter habe man das Wormser Kämmerergeschlecht von Heribert abgeleitet, bedarf zumindest der Einschränkung, daß schriftliche Zeugnisse hierfür erst aus dem späten 15. Jahrhundert stammen und in der Mehrzahl der frühen Neuzeit angehören ${ }^{14}$ ). (In diesem Zusammenhang kann ich übrigens B.s Forderung nach einer Arbeit über die Grafschaft Worms im 10./11. Jahrhundert nur unterstützen.)

Auch Tatsachen und Daten aus Heriberts Leben sind teilweise irrig wiedergegeben. Heribert starb nicht am 17. April 1021 (S. 41); des Oberhirten Todesdatum ist ider 16. März 1021 - so auch Oediger (Reg. n. 682), auf den er sonst stets verweist. Im Anschluß daran ist die Rede von der Kirche der Abtei Deutz, einer Stiftung des Erzbischofs: "J ü $\mathrm{g} s \mathrm{t}$ fand man einen bisher unbekannten ovalen Zentralbau des 11. Jahrhunderts unter den späteren Wandlungen der Kirche Heriberts" (S, 41 - Hervorhebung von mir). Doch bereits seit den Freilegungen von Wolf (um 1880), Schmitt (1909), Fremersdorff, Vogts/Haberey, und Tholen (1927 bis 1938) kennen wir diese Zentralanlage recht genau, Prechts Ausgrabungen (1971/2) bilden also nur den vorläufigen Abschluß einer längeren Folge ${ }^{15}$ ). Wieso steht der Bau in der Tradition von Kirchen "des ersten nachchristlichen Jahrhunderts, die in St. Gereon zu Köln eine Spielart haben" (S. 41 - Hervorhebung von mir)? Aachen als architektonisches Vorbild (Verbeekl) wie "ideologisches Modell" wird nicht erwähnt. Es finden sich überhaupt mehrfach solche baugeschichtlichen Exkurse, die nur entfernt im Zusammenhang mit dem Thema stehen. So ist $\mathrm{z}$. B. unvermittelt vom Dombau des. Wormser Bischofs Burchard I. (S. 52) oder von Heriberts (angeblicher) Anlage eines "strategischen Gegenpol[s] zu Deutz", nämlich dem Kölner Apostelnstift, 'die Rede (S. 53).

$\mathrm{Ob}$ der Erzbischof "sicherlich" vor 1047 kanonisiert war, erscheint mir ebenso fragwürdig ${ }^{10}$ ) wie der wiederholte Hinweis auf die "große Zuverlässigkeit" der Vita Heriberti aus der Feder des Deutzer Scholasters Lantbert von Lüttich, dessen Werk ganz andere Absichten als die Vermittlung genauer historischer Informationen verfolgte. (Ruperts Uberarbeitung ider Vita wird

\footnotetext{
13) Ebdt., S. 46-53.

10) Ebdt.; S. 48 f.

15) Ebdt., S. 284.

16) Vgl. Rhein. Vierteljahrsblätter 40 (1976), S. 46-71.
} 
übrigens nie erwähnt.) ${ }^{17}$ ) Weiterhin soll Koch (gemeint ist $\mathrm{L}$. Korth) einer "salisch-capetingischen" Familienzugehörigkeit Heriberts das Wort geredet haben; an der zitierten Stelle steht indes nur, Heribert sei der "Sohn des Grafen Hugo von Worms" ${ }^{18}$ ).

Dieser Graf Hugo wird von B. in die Nähe der Etichonen gerückt; doch ist der Name auch im Hause Vermandois bekannt. Schon im 18. Jahrhundert glaubte der Zweibrückener Rektor und Historiograph Georg Christian Crollius, in ihm einen Sohn aus der Ehe Udos mit der Vermandoiser Grafentochter, mithin einen Bruder Heriberts von der Wetterau, ausfindig gemacht zu haben ${ }^{19}$ ). Uber die zugegeben an recht entlegenem Ort und vor langer Zeit erschienenen Salierstudien von Crollius hinaus hätte $B$. allerdings weitere nützliche Auskünfte für sein Thema der Würzburger Bischofsreihe von A. Wendehorst entnehmen können, der auf die Abstammung von Heriberts bischöflichem Bruder Heinrich (995-1018) eingeht ${ }^{20}$ ). Das gilt auch für Heidingsfelders Regesten der Bischöfe von Eichstätt, unter denen sich zwei Verwandte des Kölners, Heribert (1022-1042) und Gezemann (1042), finden, ferner für K. F. Werners Arbeit über die Nachkommen Karls d. Großen zum Bereich Konradiner-Vermandois sowie für

17) $\mathrm{Zu}$ Lantbert: H. Müller, S. 3-20 - Ruperts Fassung liegt in einer neuen Edition vor: Rupert von Deutz, Vita Heriberti. Kritische Edition mit Kommentar und Untersuctiungen von Peter Dinter, Bonn 1976 (= Veröffentlichungen des Histor. Vereins f. d. Niederrhein 13).

18) Leonhard Korth, Die Patrocinien der Kirchen und Kapellen im Erzbistum KöIn, Düsseldorf 1904, S. 81 f.

10) Crollii Georg. Christ. observationes de diversis recentiorum erroribus in struenda maiorum familiae Salicae Wormatiensis s. Spirensis serie admissis, rectiusque ea a familia Loganensi Conradi I regis discernenda, quas praemittit probationibus Salicis, in: Acta Academiae Theodoro- Palatinae, t. VI (Historicum), Mannheim 1789, S. 160, 152 f.i vgl. aber S. 286.

${ }^{20}$ ) Er heiBt natürlich nicht ebenfalls Heribert (B. so S. 47) und ist auch nicht "stets auf der Seite des Kölner Bruders" (S. 48) zu finden; man denke nur an die Ereignisse des Jahres 1002, als der Würzburger sich in der Frage der Thronfolge auf die Seite Heinrichs IV. von Baiern, Heribert auf die des Schwabenherzogs Hermann II. stellte: $H$. Müller, S. 74 f., 94, 147 A. 244. 
die Dissertation von J. Milz über das Kloster Deutz im Mittelalter und anderes mehr ${ }^{21}$ ). Wenig fundiert wirken endich auch die Aussagen zu Heriberts Brüdern Reinmar und Gezemann ${ }^{22}$ ).

II.

Doch zurück zum weiteren Gang von B.s Arbeit: Der abgebrochenen Erörterung einer möglichen Zugehörigkeit des Erzbischofs zu den Konradinern folgt die Auflistung einiger frühmittelalterlicher Träger des Namens Heribert im Westen des Reiches (S. 44 bis 46). Dabei tauchen sämtliche Heriberte aus dem Index von Oedigers Regestenwerk aufl Schenken z. B. ein Heribert und sein Bruder Gerung Ende des 11. Jahrhunderts der Abtei Deutz Besitz im Siegerland (Rhein. Urkundenbuch I, n. 142), so treten wir schon in den "rechtsrheinisch-westfälischen Umkreis" (S. 46) der Heribertiner. Und dazu paßt die Intervention ides Kanzlers ${ }^{23}$ ) in einer

21) a) Alfred Wendehorst, Das Bistum Würzburg, T. 1: Die Bischofsreihe bis 1254 , Berlin 1962 (= Germania Sacra N. F. 1: Die Bistümer der Kirchenprovinz Mainz - Das Bistum Würzburg, T. 1), S. 74-88;

b) Die Regesten der Bischöfe von Eichstätt, bearb. v. Franz Heidingsfelder, Erlangen (1915-) 1938 ( Veröffentlichungen der Gesellschaft für fränkische Geschichte, VI. Reihe, 1), n. 162-181;

c) Karl Ferdinand Werner, Die Nachkommen Karls des Großen bis um das Jahr 1000 (1-8. Generation), in: Karl der Große. Lebenswerk und Nachleben, Bd. 4: Das Nachleben, Düsseldorf (1967), S. 403-484;

d) Joseph Milz, Studien zur mittelalterlichen Wirtschafts- und Verfassungsgeschichte der Abtei Deutz, Köln 1970 (= Veröffentlichungen des Köln. Geschichtsvereins, Bd. 30).

${ }^{22}$ ) a) Den lediglich im Deutzer Nekrolog erwähnten und ansonst unbekannten Reinmar sieht B. als "Heriberts jenseits der Alpen wirkenden Bruder" an; er bringt ihn mit einem Bericht des Bischofs (B.: Papstes) Leo von Vercelli an Kaiser Heinrich II. aus dem Jahre 1016 in Verbindung, nach dem der Kölner und sein Würzburger Bruder eine Nichte Reinmars Tochter?! - mit einem oberitalienischen Reichsfeind zu verheiraten gedachten. Für eine solche Vermutung bieten die Quellen nicht den geringsten Anhalt. - S. Hermann Bloch, Beiträge zur Geschichte des Bischofs Leo von Vercelli und seiner Zeit, in: NA 22 (1897), S. 11 bis 136; H. Müller, S. 178-184.

Zur möglichen Identität Reinmars mit einem im Hunsrück als Kapellenstifter zwischen 1011 und 1018 belegten "Reginmarus marchio" s. H. Müller, S. 308 A. 11. Auch hier äußert B, gewagte Vermutungen (Zusam. menhang mit dem Stift Ravengiersburg?).

b) Gezemann, im Deutzer Nekrolog am 4. März genannt (nicht am 2. März, wie B. aus Oediger, Reg.n. 562, übernimmt), war kein geistlicher Bruder Heriberts, sondern hatte wahrscheinlich die Grafschaft im unterfränkischen Werngau inne: $H$. Müller, S. $44 \mathrm{f} ., 68,74,308 \mathrm{~A} .11$.

$\left.{ }^{23}\right)$ Kanzler wurde Heribert nicht "vor $994^{\prime \prime}$, sondern Otto III, ernannte ihn Ende Sept. 994 auf dem Reichstag zu Sohlingen im Solling zum Kanzler für Italien; die deutsche Kanzlerwürde übernahm er Ende 998: H. Müller, S. 88 ff., $122 \mathrm{ff}$. 
Schenkung Ottos III. von 995 (D OIII 184) an (den später angeblich von Heribert in sein Amt lancierten Wormser Bischof) Burchard über fünf Hufen zu Her(i)bertshausen, das B. in die Nähe von Laasphe (b. Siegen) verlegt. Doch diese Ortsangabe ist bis heute ungeklärt, wie bei Boos, Uhlirz, Dietrich oder Metz nachzulesen ist ${ }^{24}$ ).

B. selbst spricht in diesem Zusammenhang einmal von "namensmäßiger Freibeuterei" (S. 46) - warum unternimmt er denn solche Abenteuerfahrten? Angesichts der Beliebtheit des Namens Heribert warnt er davor, alle dessen Träger agnatisch sammeln zu wollen, um's dann doch zu tun. Da soll doch manches anklingen, Assoziationen sollen geweckt werden, konkrete Stellungnahmen unterbleiben indes. Anstöße, Anriße $\left(_{n}\right.$ Man hat dem nachzugehen") - aber keine Ausführung ${ }^{25}$ ). Dabei läßt sich einiges recht einfach konkretisieren: Wenn z. B. der Taufname Gregors V., Brun, noch ungeklärte ottonische Beziehungen "andeuten" (S. 48) soll, so ist schlicht auf des Papstes Großmutter Liudgard, eine Tochter Ottos d. Großen, zu verweisen.

\section{III.}

Im dritten Teil seiner Ausführungen versucht B. nun, die Vorfahren des Wormser Dompropstes und KöIner Erzbischofs aus der weiblichen Linie in das Geflecht der von ihm postulierten Heribertinerdynastie einzubeziehen (S. 47-53). Ich stimme mit ihm darin überein, daß die Kognaten in der Tat größere Aufmerksamkeit verdienen, als ihnen bisher zuteil wurde, pumal Lantbert und Rupert sich ihnen ungleich ausführlicher als den Agnaten widmen. Ein wichtiges Zentrum der Heribertiner bildete das Kloster Gorze, dem ein Harbertus 987 "ob salutem animarum tam mee quam etiam parentum meorum" eine Schenkung in Vic s/Seille (süidöstl. von Château-Salins, Dép. Moselle) zukommen ließ ${ }^{26}$ ). Im Gegen-

24) Näheres bei H. Müller, S. 65 A. 103.

25) Ein typisches Beispiel hierfür ist auch die angebliche Verwendung Heriberts für das Kölner Ursulakloster (sic) bei Otto III. (S. 44, 51), vorbereitet u. a. durch den Hinweis, in einer Urkunde des Kölner Oberhirten Wichfried für das Stift aus dem Jahre 945 tauche auch der Name eines erzbischöflichen Kanzlers Heribert auf. In Wirklichkeit ist überhaupt keine Förderung der Kanonissen durch Heribert bezeugt; vgl. Gertrud Wegener, Geschichte des Stiftes St. Ursula in Köln, Köln 1971 ( $=$ Veröffentlichungen des Köln. Geschichtsvereins, Bd. 31).

26) Cartulaire de l'abbaye de Gorze. Ms. 826 de la bibliothèque de Metz publ. par A. d'Herbomez (= Mettensia II/Mémoires et documents publ. par la Soc, nat, des antiquaires de France. Fondation Auguste Prost), n. 118 - Für B. beweist das Schenkungsmotiv, daß beide Eltern vor 987 verstorben waren (S. 44, 49)! 
satz zu Oediger, der auf. diese Urkunde nur kommentarlos in einer Anmerkung zu Reg. n. 565 verweist, setzt B. den Donator mit dem späteren Erzbischof gleich, da in der Zeugenliste des Dokuments ein Tieduinus - Heriberts Mutter hieß Tiedwidis - und ein Raimarus - nach dem Deutzer Nekrolog hatte er ja einen Bruder dieses Namens - genannt werden. Zudem erhielt bereits 890 ein in Vic begüterter Erembertus von der Abtei an diesem Ort weiteren Besitz ${ }^{27}$ ). Doch ist eine Gleichsetzung allein auf Grund dieser Indizien möglich? Warum erscheint keiner der anderen Mitglieder aus der Familie des Erzbischofs - weit mehr als zehn sind quellenmäBig gesichert - im Gorzer Chartular? Wäre nicht in der Zeugenliste in irgendeiner Form ein Hinweis auf die Verwandtschaft zu erwarten? Und warum taucht Tieduinus als Zeitgenosse Heriberts weder in der Vita Lantberts noch im Deutzer Nekrolog wie die anderen Angehörigen auf? Kann man nur auf Grund dreier Namen eine Familienzusammengehörigkeit konstruieren und dabei die Uberfülle des onomastischen Materials in den Gorzer Urkunden außer acht lassen? (Die Beziehungen zwischen der Erembert-Familie und Sponville bei Briey sind B. im übrigen entgangen ${ }^{28}$ ).) Die wenigen weiteren von B. zitierten Stücke - darunter zwei Diplome Ottos d. Großen - stehen meines Erachtens in keinem erkennbaren Zusammenhang mit seiner These. Und warum tritt der Erzbischof nicht als Gönner der Abtei Prüm in Erscheinung, obwohl das Eifelkloster doch ein weiteres Heribertinerzentrum darstellt? Ist es statthaft, mit der Bemerkung "Die Relativität von Stammesbezeichnungen in dieser Zeit steht dahin" (S. 52) die eindeutige Aussage des - zuvor ausdrücklich als zuverlässig apostrophierten - Lantbert, Tiedwidis entstamme einem alemannischen Geschlecht, abzutun? ${ }^{29}$ ) Da scheint mir die von $B$. selbst ausgelegte, aber nicht weiter verfolgte Spur in den Südwesten und Süden des. Reichs ("Reginbalde") wesentlich verheiBungsvoller - dem wäre in der Tat nachzugehen.

17) Ebdt., n. 81.

28) Ebdt., n, 67, 89

29) "mater vero Tiedwidis, alto Alemanniae comitum insignis pro. fluxit a sanguine" ${ }^{\mu}$ Vita Heriberti archiep. Coloniensis auctore Lantber. to, ed. G. H. Pertz, in: MGH SS 4, Hannover 1841 (ND 1963), c, 1 (= S. 741) - Vgl. Rupert, c. $3(=$ p. Dinter, S. 35): "genus eius maternum de fonte Alemannico derivatum fuit". 
Im Zusammenhang mit der Mutter taucht wieder ein typischer Hinweis auf: "Nach Heriberts, des Kölner Metropolitaninhabers, Regierung erscheint der Kölner Suffragan Tiedwin, Dietwin, 1047 bis 1075 als Bischof von Lüttich" (S. 50) ${ }^{30}$ ). Aufs neue statt genealogischer Linien nur vage Assoziationen; daß Heribert bereits 1021 starb und Heinrich II. die Besetzung der rheinischen Stühle einem gründlichen Revirement unterzog, bleibt ungesagt. Als weiteres und letztes Beispiel solcher "Assoziationsketten" sei die Liste von Trägern des Namens Wichmann im Rahmen von B.s jüngster Untersuchung zu Heribertinern und Norbertinern angeführt, welche den Stifter der Praemonstratenser ebenfalls diesem Geschlecht zuondnen will und auch dem Stift Xanten entsprechende Aufmerksamkeit widmet. Ein Kölner Scholaster Wichmann taucht 1072 (?) in einer Schenkung des Erzbischofs Anno II. für das Kloster Groß St. Martin auf. "Dabei beruft man sich u. a. auf Schenkungen Erzbischofs Heriberts an seine Deutzer Abtei. In Xanten werden schon 1083 Reliquien des heiligen Heribert ver-

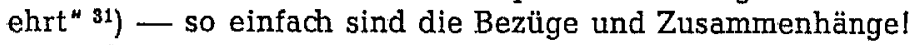

Und bald werden die Heribertiner weiteren Zuwachs erhalten; denn B. kündigt an, daß er noch "über die Anfänge der Luxemburg und heribertinischen Verbindungen" zu handeln gedenkt ${ }^{32}$ ), er also offenbar auch das erste Luxemburger Grafenhaus in seine genealogischen Konstruktionen einbeziehen will. Doch ist hierzu im Rahmen einer auf die Kritik der Ausführungen zur Familie des Erzbischofs Heribert beschränkten Miszelle nicht Stellung zu nehmen, allerdings kann ich meine Skepsis gegenüber dieser sich kontinuierlich ausweitenden "Superfamilie", deren Existenz bisheriger Forschung verborgen geblieben sein soll, nicht verhehlen. Eine Auseinandersetzung von Sachkennern wie K. F. Werner oder E. Hlawitschka mit dem Gesamtkomplex wäre erwünscht und sicher von Nutzen.

Abschließend sei die Gelegenheit für einen eigentlich nicht zum Thema gehörenden Hinweis genutzt, der aber den Erzbischof und die kölnische Kunstgeschichte betrifft. B. erwähnt die Person des

30) Wohl allein auf Grund dieser beiden Pontifikate trifft er in seiner jüngsten Arbeit die Aussage, daß sich im 10./11. Jh, das Schwergewicht des heribertinischen Stammes nach Lüttich und Köln verschoben habe: Die Familienbeziehungen des heiligen Norbert, S. 60.

si) Ebdt., S. 54 - Der Scholaster heiBt Wichmarus, und Annos II. Urkunde bezieht sich im übrigen natürlich auf eine Schenkung Heriberts an die Abtei Groß St. Martin und nicht an das Kloster Deutz: Leonhard Ennen/Gottfried Eckertz, Quellen zur Geschichte der Stadt Köln, Bd. 1, Köln 1860, n. 25.

s2) Die Familienbeziehungen des heiligen Norbert, S. 47; vgl. S. 60 A. 153 . 
Kölner Stiftsherrn und Scholasters Heribert von St. Aposteln, der sein Kanonikat aufgab, um von 1129 bis zu seinem Tode am 25. Mai 1150 als erster Propst der Praemonstratenserabtei Knechtsteden vorzustehen ${ }^{33}$ ). Ob der Heribertskelch in St. Aposteln, bisher stets mit dem Kölner Erzbischof als angeblichem Stiftsgründer in Verbindung gebracht, nicht eine Dedikation jenes Stiftsherrn und Propstes ist ${ }^{34}$ )? Diese Möglichkeit, die mir der ehemalige Pfarrer von St. Aposteln, Prof. Dr. Th. Schnitzler, andeutete, scheint in sich wesentlich stimmiger und naheliegender als die bisherigen, oft recht "gewollten "Interpretationen. Doch bliebe das Problem, daß der Kelch in seiner heutigen Gestalt erst in der ersten Hälfte des 13. Jahrhunderts gefertigt wurde; indes sind nach Schnitzler Umarbeitungen einer früheren "Grundform" nicht auszuschließen.

33) Ebdt., S. 50 f. - Vgl. Fundatio Knechtstedensis, ed. Wilhelm Gosses 4 - Gottfried Bitter, in: AHVN 165 (1963), S. 57, 59, 61 (1132-1150) - Ehlen, Die Prämonstratenser-Abtei Knechtsteden. Geschichte und Urkundenbuch, Köln 1904, S. 23 f. - Norbert Backmund, Monasticon Praemonstratense, t. I, Straubing 1949, S. 175 f. - Wilhelm Gosses, Knechtsteden und der deutsche Adel, Diss. Köln (Masch.-schr.) 1941, S. 3*-5* (Dort zur Amtszeit 1129-1150) - Ders., Zur Chronologie der Knechtstedener Pröpste und Abte, in: AHVN 149/150 (1950/1), S. 11 (Dort ebf. zur Amtszeit 1129-1150), 28.

34) Rhein und Maas. Kunst und Kultur 800-1400, Bd. 1, Köln 1972, S. 323 - Die Zeit der Staufer. Geschichte-Kunst-Kultur, Bd. 1, Stuttgart 1977, S. 428 f. - Weitere Literatur ebdt. und bei H. Müller, S. 270 A. 290. 